\title{
Phenomenological approach to the analysis of the polarization effects in fiber optic interferometers
}

\author{
J.L. Arce-Diego*, M.A. Morante Rábago, F.J. Madruga Saavedra, J.M. López-Higuera \\ Photonics Engineering Group - TEISA Department- University of Cantabria \\ Avda. los Castros s/n, E-39005 Santander (Spain)
}

\begin{abstract}
Using the scattering matrices and the graph theory, three basic structures of all-fiber interferometers, Mach-Zehnder, Michelson, and Sagnac have been analysed. The influences that, the couplers with losses and constant coupling polarization dependent, and the birefringent of the optical fibers, both used for making these optical systems, introduce in the performances of the three interferometers, are studied and compared.
\end{abstract}

Keywords: Fiber optic interferometer, polarization, scattering matrix, graph theory.

\section{INTRODUCTION}

The scattering matrix formalism in connection to the graph theory form an interesting method to describe the polarisation effects, in a phenomenological approach, on fiber optic interferometers. As an extension of the Jones calculus, the scattering matrix permits to keep the phase information, which is the basic in these optical systems. Furthermore, it makes possible to get a clear and explicit description of each of the individual elements that compound the network on the output signal. The graph theory allows us to define all the possible paths between any input and output port, for both, the signal and the reference arms of the interferometer ${ }^{1}$. It makes possible the systematic achievement of the Jones vectors in any output port of the optical fiber interferometer. In this communication, using the scattering matrices and the graph theory, the features of polarization of the couplers, with polarization dependent constant coupling and losses, and the optical fibers, used for making of this type of systems, can introduce in the performances of three basic structures of interferometers, MachZehnder, Michelson, and Sagnac, are studied and compared.

\section{MATRICIAL MODELLING OF ALL-FIBER INTERFEROMETERS}

The features of polarization of the couplers and the optical fibers used for making of this type of systems can introduce important modifications in the performances of the interferometer. In order to study in an appropriate way the characteristics of polarization in interferometers, the fibre of its signal arm will be modelled using the Kapron and Friedman rule equivalence ${ }^{2,3}$, by means of the Jones matrix $\mathbf{H}_{\mathbf{s}}=\mathbf{R}(\Omega) \mathbf{L}(\delta) \mathbf{R}(\theta)$. Any optical fiber with perturbations can be always modelled with three basic discrete elements: rotation with $\Omega$ axes, a linear phase retarder of retardation $\delta$, and a circular retarder with a rotation angle $\theta$. While the fiber of the reference arm, will be a non perturbed fiber with the Jones matrix (JM) of a constant phase retarder $\mathbf{H}_{\mathbf{r}}=\mathbf{D}(\beta \mathrm{L})$. The phase transducer with retard $\phi$ is defined by means of the matrix of the phase retarder $\mathrm{D}(\phi)$.

The couplers are polarization maintaining with coupling constants $\mathrm{K}_{\mathrm{x}}$ and $\mathrm{K}_{\mathrm{y}}$, and polarization dependent losses $\gamma_{\mathrm{x}}$ and $\gamma_{\mathrm{y}}$ ${ }^{4,5}$, and its scattering parameters $\mathrm{C}_{s}$ and $\mathrm{C}_{d}$ are given by the equation

$$
S_{31}=S_{42}=C_{d} \quad S_{41}=S_{32}=C_{s}
$$

Where

$$
\mathbf{S}_{31}=\left[\begin{array}{cc}
\sqrt{\left(1-K_{x}\right)\left(1-\gamma_{x}\right)} & 0 \\
0 & \sqrt{\left(1-K_{y}\right)\left(1-\gamma_{y}\right)}
\end{array}\right] \quad \mathbf{S}_{41}=\left[\begin{array}{cc}
j \sqrt{K_{x}\left(1-\gamma_{x}\right)} & 0 \\
0 & j \sqrt{K_{y}\left(1-\gamma_{y}\right)}
\end{array}\right]
$$

In order to model the interference, the intensity of the optical radiation at the output of each of the ports is dependent on the above mentioned parameters, and will be given by:

$$
\begin{aligned}
& I_{k}=\mathbf{E}_{k}^{\oplus} \cdot \mathbf{E}_{k}=\mathbf{E}_{i}^{\oplus} \cdot \mathbf{S}_{k i}^{\oplus} \cdot \mathbf{S}_{k i} \cdot \mathbf{E}_{i} \\
& \cdot \text { Tel.: +34-942-201545; Fax: +34-942-201873 e-mail: jlarce@teisa.unican.es }
\end{aligned}
$$


Where $\mathrm{i}, \mathrm{k}$ denote the output and the input ports, respectively, the $\oplus$ superscript denotes the Hermite conjugation, and $\mathbf{S}_{\mathrm{k}}$ is the scattering parameter that relates the $\mathrm{i}$ and the $\mathrm{k}$ ports. This parameter will be defined, in general, as the adition of two $J \mathrm{M}$, one describes the elements associated with the reference path $\mathbf{J}_{\mathrm{rkj}}$, and the other with the signal path $\mathbf{J}_{\mathrm{slj}}$ :

$$
\mathbf{S}_{k i}=\mathbf{J}_{s k i}+\mathbf{J}_{r k i}
$$

Following the Eq 3 and 4 a general expression of the output intensity $I_{k}$ in a certain output port of the interferometer can be obtained:

$$
I_{k}=A_{k i}+B_{k i} \cdot V_{k i} \cdot \cos \left(\phi^{\prime}-\phi_{k i}\right)
$$

where the $A_{k j}$ y $B_{k i}$ parameters are dependent on the couplers and the input-output configuration. The scale factor or visibility, $\mathrm{V}_{\mathrm{k}}$, is directly proportional to the system sensitivity and can be defined in the following way

$$
V_{k i}=a b s\left(v_{k i}\right) \quad \phi_{k i}=\arg \left(v_{k i}\right)
$$

with

$$
v_{k i}=\mathbf{E}_{i}^{\oplus} \mathbf{H}_{r k i}{ }^{\oplus} \mathbf{H}_{s k i} \mathbf{E}_{i}
$$

where $\mathbf{H}_{\mathrm{rdi}}$ y $\mathbf{H}_{\mathrm{sk}}$ are $\mathrm{JM}$ that describe the reference and signal path, respectively, only considering the polarization effects of the fibers, that is, they don't take into account the coupler effects and the phase transducter. Finally, the phase coefficient $\phi^{\circ}$ is an addition of the phase perturbations generated in a certain configuration.

\section{THE MACH-ZEHNDER INTERFEROMETER}

The two-beam interferometer Mach-Zehnder is an in-directional, the propagation takes place only in one forward way, and unbalanced interferometer, and it is shown in the Fig. 1. It is compound by two couplers and two sections of fibers with lengths $\mathrm{L}_{1}$ and $\mathrm{L}_{2}$. When an optical signal is introduced at the input, using port 1 or port 2 , the first coupler acts as a beam splitter dividing the signal into $\mathrm{L}_{1}$ and $\mathrm{L}_{2}$ arms. Finally, the second coupler combines the output signals of both arms, making possible to extract the resultant signal through port 3 or 4.

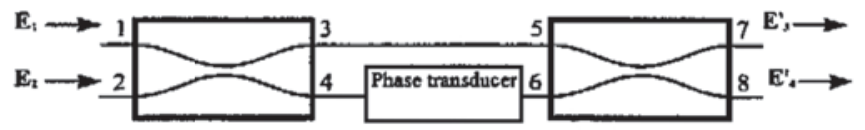

Figure 1: Mach-Zehnder interferometer.

By using the topological representation of the fibers and the couplers and applying the rule of the graph connection ${ }^{1}$, it is possible to obtain the graphs that represent the Mach-Zehnder interferometer for all the output-input configurations ( see Fig. 2 ), and using the reduction rules the four scattering parameters that characterise this structure are obtained.

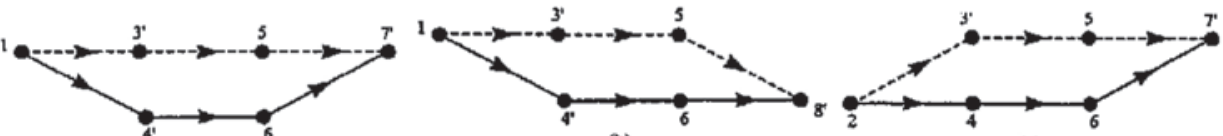

(n) (b)

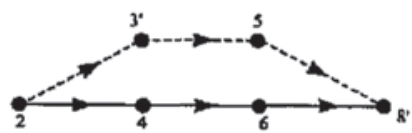

(ब)

Figure 2: Graphs associated to the Mach Zehnder interferometer for the obtention of the scattering parameters in transmission: (a) $\mathrm{S}_{31}$, (b) $\mathrm{S}_{41}$, (c) $\mathrm{S}_{32}$, (d) $\mathrm{S}_{42}$. Reference path with dashed lines and signal path with solid lines.

These scattering parameters $\mathbf{S}_{\mathrm{ki}}$ have the general expression given in the Eq. (4), where the JM for the possible input-output combinations are given in the following table:

\begin{tabular}{|l|c|c|}
\hline$(K, I)$ & $\mathbf{J}_{\mathrm{RKI}}$ & $\mathbf{J}_{S K I}$ \\
\hline$(3, I)$ & $\mathbf{C}_{d} \mathbf{D}\left(\Delta_{1}\right) \mathbf{C}_{d}$ & $\mathbf{C}_{s} \mathbf{D}\left(\Delta_{2}\right) \mathbf{R}(\Omega) \mathbf{L}(\delta) \mathbf{R}(\theta) \mathbf{C}_{s} \mathbf{D}(\phi)$ \\
\hline$(3,2)$ & $\mathbf{C}_{d} \mathbf{D}\left(\Delta_{1}\right) \mathbf{C}_{s}$ & $\mathbf{C}_{s} \mathbf{D}\left(\Delta_{2}\right) \mathbf{R}(\Omega) \mathbf{L}(\delta) \mathbf{R}(\theta) \mathbf{C}_{d} \mathbf{D}(\phi)$ \\
\hline$(4,1)$ & $\mathbf{C}_{s} \mathbf{D}\left(\Delta_{1}\right) \mathbf{C}_{d}$ & $\mathbf{C}_{d} \mathbf{D}\left(\Delta_{2}\right) \mathbf{R}(\Omega) \mathbf{L}(\delta) \mathbf{R}(\theta) \mathbf{C}_{s} \mathbf{D}(\phi)$ \\
\hline$(4,2)$ & $\mathbf{C}_{s} \mathbf{D}\left(\Delta_{1}\right) \mathbf{C}_{\mathbf{s}}$ & $\mathbf{C}_{d} \mathbf{D}\left(\Delta_{2}\right) \mathbf{R}(\Omega) \mathbf{L}(\delta) \mathbf{R}(\theta) \mathbf{C}_{d} \mathbf{D}(\phi)$ \\
\hline
\end{tabular}

Table 1: Jones Matrix of the signal and reference arms for the Mach-Zehnder interferometer. 
Where $\Delta_{i}=\beta . L_{i}$ is the delay due to the propagation in the fiber of length $\mathrm{L}_{i}$. As you can observe the differences between the topologies are only due to the couplers. The $v_{\mathbf{k}}$, parameter that doesn't consider the effects of the couplers will be the same for all of them $\left(V_{k i}=V, \phi_{k i}=\phi_{0}\right)$ and, considering that the two fibers are identical and with the same length $\left(\Delta_{1}=\Delta_{2}\right)$, it can be expressed according to Eq. 7, following the expression:

$$
v=\mathbf{E}_{\text {in }}^{\oplus} \mathbf{R}(\Omega) \mathbf{L}(\delta) \mathbf{R}(\theta) \mathbf{E}_{\text {in }}
$$

By means of the $v$ parameter it is possible to analyse the effects that any input state of polarisation (SOP) $\mathbf{E}_{\text {in }}$ may produce over the features of polarisation of the optical fiber. Being $\mathbf{E}_{\text {in }}$ defined by the azimuth $\xi$ and the elipticity $\boldsymbol{\varepsilon}$. When a linear polarizer is introduced at the input $(\varepsilon=0)$, a rotation angle $\alpha=\theta+\Omega$ without linear birefringence linear $(\delta=0)$, causes only a change in the sensitivity of the system, being independent of the azimuth of the input SOP. On the other hand, a linear birefringence introduces a sensitivity of the system to the azimuth of the input SOP. Therefore, the change of the azimuth of the output SOP, for a variable linear birefringence, also produces a modification of the $\mathrm{V}$ y $\phi$ parameters. However, in the case of an exact adjustment of the system, for example, uniform equal excitation of both modes of the fiber $(\xi=(2 n+1) \pi / 4)$, the birefringence causes a modification in the sensitivity level. If only one mode is propagated $(\xi=n \pi / 2)$ the sensitivity is constant, while the phase is directly proportional to the birefringence of the fiber, according to previous results ${ }^{6}$. By using the expressions of the Table 1 and the Eq.3 it is possible to get the irradiance in each of the output ports, similar to the Eq. 5, with $\phi^{\prime}=\phi$. This function depends on the characteristic parameters of the network. Fig. $3 \mathrm{a}$ shows the irradiance versus the linear birefringence of the signal arm when the ligth gets into port 1 , and gets out of port 3 , assuming $\alpha=\theta+\Omega=0$. On the contrary, considering $\delta=0$, we can get a representation of the irradiance in function of the rotation of the polarization plane $\alpha=\theta+\Omega$ (see Fig.3b). In both analysis a linear SOP with azimuth $\xi=\pi / 4$ was considered. Moreover, equal couplers, lossless, and with isotropic coupling constants have been considered $\left(\mathrm{K}_{\mathrm{x}}=\mathrm{K}_{\mathrm{y}}=\mathrm{K}=0.5\right)$.

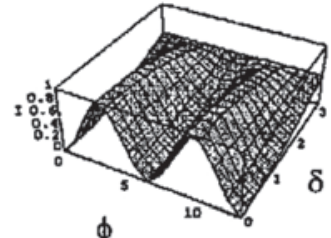

$\phi$

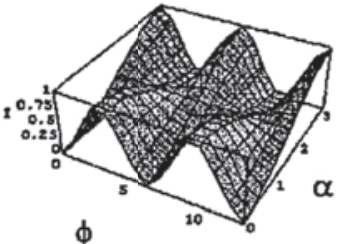

$\phi$
Figure 3. Output intensity for a) a variable lineal birefringence $\delta$ b) a variable rotation $\alpha$ of the polarization plane of the fiber. The input signal, with $\xi=\pi / 4, \varepsilon=0$, is introduced at the port 1 and is obtained at port 3. The couplers are considered both with $\mathrm{K}_{\mathrm{x}}=\mathrm{K}_{\mathrm{y}}=0.5$.

In order to obtain the variation of the output intensity in function to the coupling constants of the couplers, we take $\theta+\Omega=\alpha=0$ and $\delta=0$. In the Fig. 4 a representation of the intensity in function of the coupling constant $\mathrm{K}$, considering polarization independent couplers, is obtained. The $\mathrm{K}$ variation produces changes in the visibility of the system, getting the maximum visibility for $\mathrm{K}=0.5$. In the case of anisotropic couplers, Fig. $4 \mathrm{~b}$ shows the results obtained, in which the intensity appears in relation to $\mathrm{K}_{\mathrm{x}} / \mathrm{K}_{\mathrm{y}}$. As expected, the maximum visibility is reached for $\mathrm{K}_{\mathrm{x}}=\mathrm{K}_{\mathrm{y}}=0.5$. Fig. 5 a shows the effects of the isotropic losses of the couplers. Fig. $5 \mathrm{~b}$, represents the variation of the output intensity in relation to $\gamma_{\mathrm{y}} / \gamma_{\mathrm{x}}$, being $\gamma_{\mathrm{x}}=0.1$.
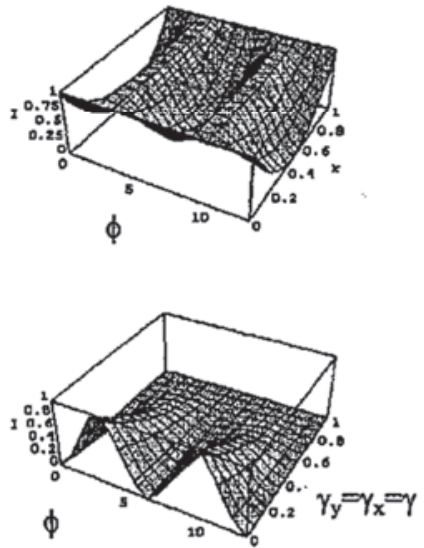
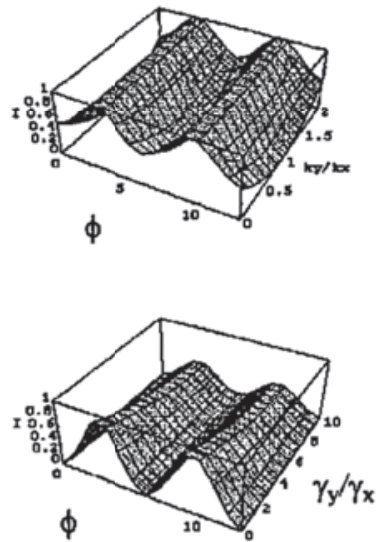

Figure 4. A representation of the output intensity, with $\delta=0$ and $\alpha=0$ for a) a constant coupling $\mathrm{K}$ variable b) $\mathrm{K}_{\mathrm{y}} / \mathrm{K}_{\mathrm{x}}$ variable. The input signal, with $\xi=\pi / 4, \varepsilon$ $=0$, gets into port 1 and gets out of port 3 .

Figure 5. A representation of the output intensity, with $\delta=0$ and $\alpha=0$, for a) coupling losses $\gamma_{x}=\gamma_{y}=\gamma$ variable b) $\gamma_{y} / \gamma_{x}$ variable.. The input signal, with $\xi=\pi / 4, \varepsilon$ $=0$, gets into port 1 and gets out of port 3 .

If the signal is obtained through port 4, there will be a shift of $\pi$ rad due to the action of the couplers. In relation to the other parameters the situation is similar to the previously obtained in the case of the port 3 . 


\section{MICHELSON INTERFEROMETER}

The Michelson interferometer is a counter-directional and unbalanced interferometer and its structure is represented in Fig.6 As it can be observed it is composed by a directional coupler, two optical fibers with length $L_{1}$ and $L_{2}$, and two isotropic reflectors $T_{1}$, and $T_{2}$
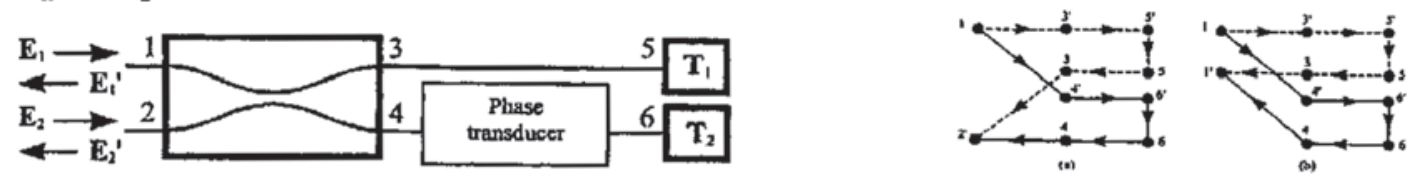

Figure 6: Michelson interferometer scheme and its associated graphs to the forgetting the scattering parameters: (a) $S_{21}$, (b) $S_{11}$. Reference path with dashed lines and signal path with solid lines.

In this interferometer, the reflectors make the signal to be reflected and finally be combined into the coupler. As the waves travel in both directions this structure is counterdirectional. It is assumed that the two reflectors have the same $\mathrm{JM}^{?}$. If the two fibers are identical and of the same length $L$, the scattering matrix of the interferometer is symmetric. $S_{11}$ and $S_{21}$ parameters are obtained by using the graph reduction rules and they can be expressed according the Eq.4 by means of the JM given in the following table:

\begin{tabular}{|c|c|c|}
\hline$(k, i)$ & $\overline{I_{\mathrm{kj}}}$ & $\overline{\bar{S}_{\text {ati. }}}$ \\
\hline$(1,1)$ & $C_{s} D\left(2 \Delta_{1}\right) \mathbf{T}\left(e^{-j} \pi\right) C_{d}$ & $C_{d} \mathbf{D}\left(2 \Delta_{2}\right) \mathbf{R}^{\top}(\theta) \mathbf{L}(\delta) \mathbf{r}\left(\mathrm{e}^{j} \pi\right) \mathbf{L}(\delta) \mathbf{R}(\theta) C_{s} \mathbf{D}(2 \phi)$ \\
\hline$(2,1)$ & $C_{d} D\left(2 \Delta_{1}\right) T\left(e^{-j} \pi\right) C_{d}$ & $C_{d} \mathbf{D}\left(2 \Delta_{2}\right) \mathbf{R}^{\mathrm{T}}(\theta) \mathbf{L}(\delta) \mathbf{T}\left(\mathrm{e}^{j} \pi\right) \mathbf{L}(\delta) \mathbf{R}(\theta) C_{s} \mathbf{D}(2 \phi)$ \\
\hline
\end{tabular}

Table 2: Jones matrices of the signal and reference arms for Michelson interferometers.

As in the case of the Mach-Zehnder interferometer, the diferences between input-output configurations is only due to the couplers. The $v_{\mathrm{ki}}$ parameter, which is not dependent of the couplers, will be the same for all the configurations $\left(\mathrm{V}_{\mathrm{kj}}=\mathrm{V}\right.$, $\left.\phi_{\mathrm{ki}}=\phi_{0}\right)$ and in the case that the two fibres are identical and with the same length $\left(\Delta_{1}=\Delta_{2}\right)$, it can be expressed by (see Eq.7):

$$
v=\mathbf{E}_{i n}{ }^{\oplus} \mathbf{R}(-\theta) \mathbf{L}(2 \delta) \mathbf{R}(\theta) \mathbf{E}_{i n}
$$

By means of this expression it is possible to obtain, that the fibre acts as a linear retarder $2 \delta$ with an angle rotation of $-\theta$ respect to the $\mathbf{X}$ axis of the co-ordinate reference system in this configuration. If only a rotation of the plane of polarization $(\delta=0)$ is introduced, the visibility is 1 , and is independent of the input SOP. This configuration is insensitive to a rotation of the plane of polarization in the optical fiber, due to the light travels twice the same optical path in the fiber.

In the case that the fibre only has linear birefringence $(\theta=0)$, a variation of the system parameters with the input azimuth is produced. This structure is twice more sensitive to a change of the linear birefringence, in comparison with the MachZehnder interferometer (unidirectional), and the dependence of this sensitivity with the SOP is similar to the Mach-Zehnder interferometer. Then, the system is only dependent of the azimuth of the input radiation. If an optical signal is introduced into port 1 and comes out through port 2, with $\theta=0$, a representation of the irradiance in function of the linear birefringence $\delta$ is shown in Fig. 7b. In the case of $\delta=0$, we can obtain a representation of the irradiance in function of the rotation of the polarization plane $\alpha=\theta+\Omega$ (see Fig. 7a) In both calculus a linear SOP with azimuth $\xi=\pi / 4$, is used and $\mathrm{K}_{\mathrm{x}}=\mathrm{K}_{\mathrm{y}}=0.5$.
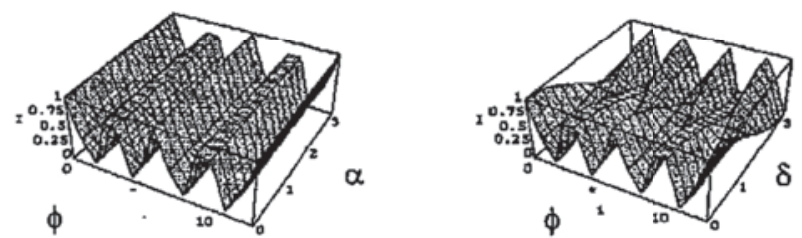

Figure 7: A representation of the output intensity for a) a variable rotation $\alpha$ of the polarization plane. b) a linear variable birefringence $\delta$. Input signal, with $\xi=\pi / 4, \varepsilon$ $=0$, gets into port 1 and gets out of port 2 . Both couplers with $\mathrm{K}_{\mathrm{x}}=\mathrm{K}_{\mathrm{y}}=0.5$

Figures 8a,b show the output optical intensity in function of the linear birefringence and the rotation of the plane of polarisation, respectively, signal input in port 1 and out in port 1 . In these figures it is possible to observe a change of $\pi / 2$ in the phase with respect to the case in which the signal gets out of the port 2. 

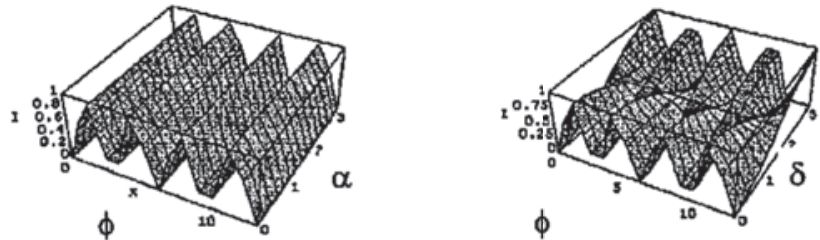

Figure 8: Output intensity signal for a) a variable polarization rotation plane $\alpha$ b) a linear variable birefringence $\delta$. Input signal, with $\xi=\pi / 4, \varepsilon=0$, gets into port 1 and gets out of port 2. Both couplers with $K_{x}=K_{y}=0.5$

The variation of the parameters of the couplers produces the same effect that for a Mach-Zehnder interferometer, that is, both the variation of the constant coupling and the effect of the losses produce the decreasing in of the system sensitivity.

\section{SAGNAC INTERFEROMETER}

This interferometer is counter-directional as the Michelson interferometer, however is balanced, because its propagation takes place in an only fiber. It only needs a coupler, and then the distribution of the output optical power is similar to the Michelson interferometer.

A scheme of the Sagnac interferometer is shown in Fig.9a, and is made of a loop of optical fibre connected to the two output ports of a directional coupler. If the optical radiation is coupled into port 1 and the coupler is a $3 \mathrm{~dB}$ coupler, ( $\mathrm{K}=0.5)$, then $50 \%$ of the input radiation traveis in a clockwise sense and the other $50 \%$, in a counterclockwise sense. Moreover, the light that gets out of port 4 undergoes a phase delay of $\pi / 2$ respect the light that gets out of port 3 . The intensity transmitted to the port 2 will be the result of the addition of an optical field which travels in a clockwise sense, with a phase $\phi$, and another optical field in a counterclockwise sense, with a phase $\phi-\pi$, being both of the same amplitude. This produces a fading in the transmitted signal and in accordance to the energy conservation principle all the intensity is reflected in port 1.
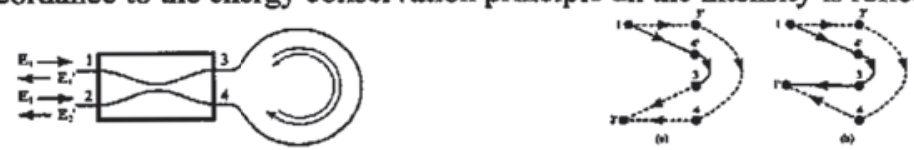

Figure 9: Sagnac interferometer scheme and its associated graphs for the obtention of its scattering parameters. (a) $S_{21}$, (b) $S_{11}$. Reference path with dashed lines and signal path with solid lines..

According to the description previous we can assume that this structure can work as a reflector with a reflectivity equal to unity. Neverthe less, this is a very idealized description, because in practice coupling ratio is not exactly $50 \%$ at the working wavelength, and there are coupling losses and birefringences in the fiber. The behaviour of the interferometer is determined by these parameters. The associated graphs of this structure in order to obtain the $S_{11}$ and $S_{21}$ parameters are shown in Fig.9b. This structure is symmetric and finally $\mathbf{S}_{22}=\mathbf{S}_{11}$ y $\mathbf{S}_{12}=\mathbf{S}_{21}$. Then, the scattering matrix has the same form of Michelson interferometer with the difference of the definition of the $\mathbf{J M}$, as is showed in following table:

\begin{tabular}{|c|c|c|}
\hline$(k, i)$ & $\overline{J_{t k i}}$ & $\mathbf{J}_{\mathrm{kg}}$ \\
\hline$(1,1)$ & $\mathbf{C}_{\mathbf{d}} \mathbf{D}(\Delta) \mathbf{I n v} \mathbf{R}^{\mathrm{T}}(\theta) \mathbf{L}(\delta) \mathbf{R}^{\mathrm{T}}(\Omega) \mathbf{C}_{\mathbf{s}} \mathbf{D}(\phi)$ & $C_{s} \mathbf{D}(\Delta) \mathbf{R}(\Omega) \mathbf{L}(\delta) \mathbf{R}(\theta) \operatorname{InvC}_{d} \mathbf{D}(\phi)$ \\
\hline$(2, I)$ & $\operatorname{CsD}(\Delta) \operatorname{Inv} \mathbf{R}^{\mathrm{T}}(\theta) \mathbf{L}(\delta) \mathbf{R}^{\mathrm{T}}(\Omega) \operatorname{CsD}(\phi)$ & $\mathbf{C}_{d} \mathbf{D}(\Delta) \mathbf{R}(\Omega) \mathbf{L}(\delta) \mathbf{R}(\theta) \mathbf{I n v C}_{d} \mathbf{D}(\phi)$ \\
\hline
\end{tabular}

Tabla 3: Jones matrices of the signal and reference arms for the Sagnac interferometer.

The Inv matrix is the coordinates inversion matrix. The $v$ parameter, see the Eq.7, is given by:

$$
v=\mathbf{E}_{i n}^{\oplus} \mathbf{R}(\Omega) \mathbf{L}(-\delta) \mathbf{R}(\Omega+\theta) \mathbf{L}(\delta) \mathbf{R}(\theta) \mathbf{E}_{i n}
$$

The Sagnac interferometer, if only a rotation of the input plane of polarization is allowed, the dependence on the parameters of the system with the input azimuth shows the same behaviour of the Mach-Zehnder interferometer. That means that the system is independent of the input azimuth. On the other hand, if only lineal birefringence exists the visibility is constant and equal to unity with $\phi_{0}=0$, so the system is not affected by a pure linear birefringence. The special characteristics of this configuration are due to the utilization of the same optical fibre for the reference and signal beams. Due to the propagation in opposite sense of both beams, the effect of the linear birefringence is eliminated. The explanation is that the two beams have the same SOP in any point of the loop with independence of the input SOP. In this interferometer, the existence of a rotation in the plane of polarization of the fiber produces the same effect in both beams. Therefore, for a rotation of $\pi / 4$, both beams are orthogonal at the output, and the sensitivity fades to zero. If the rotation angle is $\pi / 2$ (sensitivity=0 in the MachZehnder), the output beams are rotated an angle $\pi$. That is, the output beams are parallel and the sensitivity is the highest. In this case, the phase is shifted $\pi \mathrm{rad}$, by this behaviour doesn't modify the transference function of the system. If the coupler effects are not taking into account, for $\alpha=n \pi / 4$, and with independent of the input SOP, the output intensity in function of $\phi$ is 
constant and equal to 1 . However, the polarization characteristics of the coupler can introduce variations in the reflectivity, as it is observed in Fig.10a, where the output intensity is represented in function of the coupler constant $\mathrm{K}$.
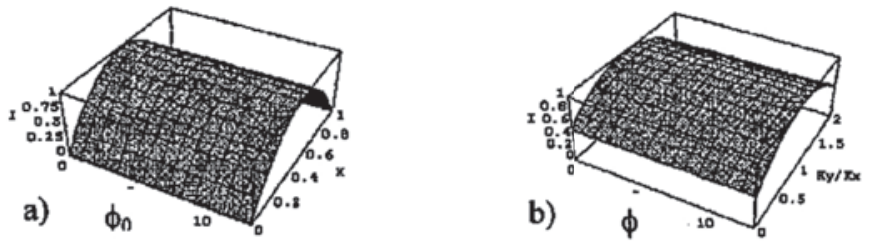

Figure 10: Output intensity, with $\alpha$ $=n \pi / 2$ for a) coupling ratio $K$ variable b) $K_{g} / K_{x}$ variable.

In Fig. 10b the effects produced by different for each polarization are shown. Likewise, in the Fig.11 the effects of the isotropic losses in the coupler are depicted, and the reflectivity decrease when the losses increased.

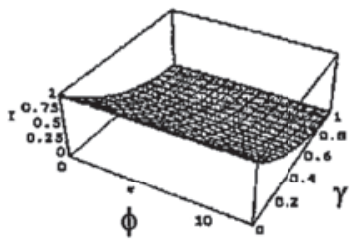

Figure 11: Output intensity vs. coupler losses.

In regars to the method of analysis that has already been presented, it is important tomention that it can be extended to study polarization effects over all types of all-fiber interferometers including optical gain along its branches.

\section{CONCLUSIONS}

A research of the behaviour with the input SOP of different types of all-fiber inteferometers has been made. By using both, the scattering matrices and the graph its scattering parameters, as well as the output intensity for each of its ports, have been obtained. These intensities are dependent on the input state of polarization, the characteristics of the couplers, and the optical fibers, which compose the interferometers. The influences, on the interference signal, of the coupling Constants and the losses of the couplers, both polarization dependent, as well as the external birefringences have been analysed. In general, we can conclude by stating that the Mach-Zenhder interferometer is the one that presents over the above mentioned influences. The Michelson one offers a better performance although it keeps a high sensitivity in relation to linear birefringence. On the other hand, the Sagnac interferometer is the one, which presents the lowest influence due to its reciprocal optical structure.

\section{ACKNOWLEDGEMENTS}

This work has been partially funded by the Spanish C.I.C.Y.T. Commission through the project TIC'98-0397-C03-02.

\section{REFERENCES}

1. Y.Weissman, Optical Network Theory, Artech House, Inc, Boston, 1992.

2. F.P.Kapron, N.F.Borelli, D.B.Keck, "Birefringence in dielectric optical waveguides", IEE Journal of Quantum Electronics, Vol.QE-8, pp 222-225, 1972.

3. Friedman, B., Principles and techniques of applied mathematics, Chapman and Hall, New York, 1956.

4. C.Chen, W.K.Burns, "Polarization characteristics of single-mode fiber couplers", IEEE Journal of Quantum Electron., Vol. $Q E-18$, pp 1589-1600, 1982.

5. Miguel V.Andrés, Kenneth W.H.Foulds, "Optical fiber resonant rings based on polarization dependent-couplers", Journal of Lightwave Technol., Vol.8, $N^{\circ} 8$, pp 1212-1220, 1990.

6. L.R. Jaroszewicz, "Polarization role in fiber optic interferometers", Proc. SPIE, vol. 3094, pp.204-217, 1997.

7. R.M.Azzam and N.M.Bashara, Ellipsometry and Polarized Light, North-Holland, Amsterdam.1987. 